\title{
Comportamento sedentário e declínio cognitivo em idosos comunitários
}

\section{Sedentary behavior and cognitive decline in community-dwelling older adults}

\author{
AUTORES \\ Priscila Resende Horacio $^{1}$ (D) \\ Núbia Carelli Pereira de Avelar ${ }^{1}$ (ID \\ Ana Lúcia Danielewicz ${ }^{1,2}$ (D) \\ 1 Universidade Federal de Santa Catarina, \\ Departamento de Ciências da Saúde, Curso de \\ Fisioterapia, Araranguá, Santa Catarina, Brasil. \\ 2 Universidade Federal de Santa Catarina, Programa \\ de Pós-Graduação em Ciências da Reabilitação, \\ Araranguá, Santa Catarina, Brasil.
}

\section{CONTATO}

\section{Ana Lúcia Danielewicz}

ana.lucia.d@ufsc.br

Universidade Federal de Santa Catarina -

Campus Araranguá, Rodovia Governador

Jorge Lacerda, 3201, Jardim das Avenidas, Araranguá, Santa Catarina. Brasil.

CEP: 88906-072.

DOI

$10.12820 /$ rbafs. $26 \mathrm{e} 0190$

\section{(cc) BY}

Este trabalho está licenciado com uma Licença Creative Commons - Atribuição 4.0 Internacional.

\begin{abstract}
RESUMO
O comportamento sedentário está associado a doenças cardiovasculares, baixa qualidade de vida e maior risco de mortalidade. Evidências sugerem que o comportamento sedentário pode contribuir para o declínio cognitivo, contudo a magnitude e a direção dessa associação não estão completamente esclarecidas. O objetivo foi avaliar a associação entre o comportamento sedentário e o declínio cognitivo em idosos comunitários. Estudo transversal com amostra probabilística e de base domiciliar, com idosos cadastrados na Atenção Básica de Balneário Arroio do Silva, Santa Catarina. O comportamento sedentário foi avaliado pela questão "tempo gasto sentado" do International Physical Activity Questionnaire,categorizado em: 0 a 3 horas; 4 a 7 horas; 8 a 10 horas; e 11 ou mais horas por dia. O declínio cognitivo foi avaliado pelo Mini Exame do Estado Mental, considerando os pontos de cortes recomendados por Brucki et al. ${ }^{14}$. Foram realizados modelos de Regressão Logística Multivariada brutos e ajustados, apresentando-se os valores de odds ratio (OR) e IC95\%. Foram analisados 308 idosos, sendo $57,8 \%$ mulheres e com média de idade 60,8 anos ( $\mathrm{DP}=7,06$ anos). A prevalência de declínio cognitivo foi $57,7 \%$, com predomínio em mulheres, menor faixa etária e piores comportamentos de saúde. O modelo final da regressão mostrou que os idosos que permaneciam 11 ou mais horas sentados por dia tiveram cinco vezes mais chances de terem declínio cognitivo (OR = 5,03; IC95\%:1,07; 23,61) quando comparados aos que permaneciam por até 3 horas por dia nesse comportamento. Concluiu-se que houve associação positiva entre maior comportamento sedentário e declínio cognitivo nos idosos avaliados.
\end{abstract}

Palavras-chave: Função cognitiva; Envelhecimento; Comportamento sedentário.

\section{ABSTRACT}

Sedentary behavior is associated with cardiovascular disease, loss of autonomy and functional independence, worse quality of life and increased mortality. Preliminary evidence supports that sedentary behavior is associated with cognitive decline, however the magnitude and direction of these associations are not completely understood. The aim of this study was to verify the association between sedentary behavior and cognitive decline community-dwelling older adults. Cross-sectional study with a probabilistic and home-based sample of older adults registered in Primary Care in the municipality of Balneario Arroio do Silva, Santa Catarina, Brazil. Sedentary behavior was assessed by the question "time spent sitting" in the International Physical Activity Questionnaire and categorized into: 0 to 3 hours; 4 to 7 hours; 8 to 10 hours; and 11 or more hours a day. Cognitive decline was assessed by the Mini Mental State Examination, considering the cutoff points recommended by Brucki et al. ${ }^{14}$. Crude and adjusted Multivariate Logistic Regression models were performed, presenting the odds ratio and 95\% CI values. A total of 308 older adults were evaluated, $57.8 \%$ of whom were female and with a mean age of 60.8 years $(S D=7.06$ years). The prevalence of cognitive decline was 57.7\%, with a predominance of women, with a lower age range and worse health behaviors. The final regression model pointed out that the older adults who remained seated 11 or more hours/day were five times more likely to have cognitive decline $(O R=5.03$; $95 \%$ CI: 1.07; 23.61) when compared to those who remained for up to 3 hours/day sitting. In conclusion, there was a positive and significate association between greater sedentary behavior and cognitive decline in the elderly evaluated.

Keywords: Cognitive function; Aging; Sedentary behavior.

\section{Introdução}

O envelhecimento é um processo complexo que ocorre de forma progressiva, com velocidade e agilidade vivenciadas de formas individuais, e que envolve declínios nas habilidades funcionais e cognitivas ${ }^{1}$. Nesse aspecto, o declínio cognitivo pode ser caracterizado pelo comprometimento de habilidades importantes, tais como memória, atenção, orientação, fluência verbal e funções executivas, o que pode evoluir para diagnósticos mais graves como as demências ${ }^{2}$.

Mundialmente as prevalências de declínio cognitivo nos idosos tendem a variar entre $5 \%$ a $30 \%$ conforme o sexo, faixa etária e gravidade da doença ${ }^{3}$. No Brasil, observam-se variações conforme as regiões, sendo 
relatado 4,9\% em idosos residentes em Pouso Alegre - Minas Gerais ${ }^{4}$, 19,1\% em idosos de Montes Claros - Minas Gerais ${ }^{5}$ e 34,1\% em idosos de Bagé - Rio Grande do $\mathrm{Sul}^{6}$. O declínio cognitivo costuma ser mais frequente em idosas, com baixa renda e escolaridade e com doenças crônicas associadas, tais como a hipertensão arterial sistêmica, a diabetes mellitus e a depressão ${ }^{7}$.

Ainda com relação aos fatores predisponentes ao declínio cognitivo, destaca-se sua associação com o comportamento sedentário, o qual pode ser caracterizado pelos comportamentos adotados nas posições sentada, reclinada ou deitada, e que não apresentam um gasto energético acima dos níveis de repouso (1,0 - 1,5 equivalentes metabólicos), como por exemplo, o uso de computador, celular ou televisão ${ }^{8}$. Infere-se que idosos que despendem mais tempo diário em comportamento sedentário têm risco aumentado de acometimentos cognitivos, quando comparados aos que não apresentam esse comportamento ${ }^{8}$. Ademais, a participação em programas de exercícios físicos regulares pode exercer efeitos preventivos ao declínio cognitivo, pois o processamento cognitivo ocorre de forma mais rápida em idosos ativos ${ }^{9}$. Destaca-se ainda, que o comportamento sedentário excessivo é frequente em idosos, os quais tendem a gastar em média 9,4 horas por dia em atividades na posição sentada, tornando-se, portanto, um fator de risco importante para o declínio cognitivo ${ }^{10}$.

Sabe-se que o comportamento sedentário está associado a diversos riscos à saúde, incluindo a ocorrência de doenças cardiovasculares, perda da autonomia e da independência funcional, pior qualidade de vida e aumento da mortalidade por todas as causas ${ }^{4}$. E, embora as evidências preliminares suportem que o comportamento sedentário esteja associado ao declínio cognitivo, ainda não estão totalmente esclarecidas a magnitude e a direção dessas associações.

Com isso, verifica-se a necessidade de estudos que evidenciem a associação do comportamento sedentário ao declínio cognitivo em idosos, visando promover maior conhecimento sobre o assunto aos profissionais da saúde, familiares e cuidadores, a fim de que esses possam estimular a redução desse comportamento e, consequentemente, a preservação da função cognitiva. Desse modo, o objetivo do presente estudo foi avaliar a associação entre o comportamento sedentário e o declínio cognitivo em idosos comunitários.

\section{Métodos}

Tratou-se de um estudo transversal, com amostra pro- babilística, de base domiciliar, realizado com idosos (60 anos ou mais), de ambos os sexos, cadastrados no Sistema de Gestão Estratégica da Saúde (SIGES) do município de Balneário Arroio do Silva, Santa Catarina.

O município está localizado na mesorregião sul catarinense. De acordo com o Censo Demográfico de $2010,15,79 \%$ da população era idosa ${ }^{11}$. O Índice de Desenvolvimento Humano (IDHM) do município para o referido ano foi 0,746 , sendo que os fatores que mais contribuíram foram a longevidade $(0,858)$, renda mensal per capita $(0,753)$ e educação $(0,642)^{12}$.

O cálculo amostral foi realizado com base no total de idosos cadastrados $(n=2.833)$ nas três Unidades Básicas de Saúde (UBS) do município (Centro = 801; Zona Nova Sul $=867$ e Erechim $=1165)$. Considerou -se prevalência para desfechos desconhecida de 50\%, nível de confiança de $95 \%$, erro amostral de seis pontos percentuais, $20 \%$ para perdas previstas e $15 \%$ para análises dos estudos de associação, estimando-se o mínimo de 302 voluntários. Consideraram-se elegíveis para compor a amostra 540 idosos, os quais foram sorteados aleatoriamente conforme sua representatividade em cada UBS: Centro = 152 idosos (28,1\%); UBS Zona Nova Sul = 163 idosos (30,2\%); e UBS Erechim = 225 idosos $(41,7 \%)$.

Foram excluídos os idosos residentes em instituições de longa permanência, que haviam mudado o endereço residencial, acamados/dependentes que não puderam responder aos questionários, e também aqueles que não foram localizados em seus domicílios após três tentativas realizadas em dias e horários distintos. Consideraram-se recusas aqueles que não aceitaram participar do estudo após receberem a visita dos entrevistadores.

A coleta dos dados foi realizada entre os meses de setembro de 2018 e setembro de 2019. Inicialmente, os idosos selecionados foram contatados via telefone e convidados a participarem do estudo, sendo então agendadas as entrevistas em seus domicílios. Após essa etapa, os demais idosos que não atenderam aos telefonemas foram visitados pelos entrevistadores, sempre acompanhados pelas Agentes Comunitárias de Saúde responsáveis pela microárea correspondente à sua residência. Cada entrevista tinha duração de 40-50 minutos.

A equipe de entrevistadores foi composta por discentes de graduação do curso de Fisioterapia e do Programa de Pós-graduação em Ciências da Reabilitação. Todos os entrevistadores foram previamente treinados em duas etapas, sendo uma expositiva dialogada sobre os objetivos de cada instrumento de coleta, e outra prá- 
tica, com finalidade de adaptação, calibração e padronização das avaliações. Ainda com o objetivo de auxiliar o trabalho de campo foi elaborado um manual para o entrevistador contendo explicações detalhadas de todos os instrumentos de pesquisa.

Os idosos participantes receberam orientações sobre os objetivos da pesquisa e assinaram o Termo de Consentimento Livre e Esclarecido. $\mathrm{O}$ estudo recebeu aprovação pelo Comitê de Ética em Pesquisa com Seres Humanos da Universidade Federal de Santa Catarina (CAAE no87776318.3.0000.0121).

O desfecho declínio cognitivo foi avaliado pelo Mini Exame do Estado Mental (MEEM), traduzido e validado para a população brasileira ${ }^{13}$. O MEEM é composto por 30 questões que avaliam as funções cognitivas como orientação temporal, orientação espacial, registro de três palavras, atenção e cálculo, recordação das três palavras, linguagem e capacidade construtiva visual ${ }^{13}$. Foi adotado o ponto de corte para classificação do declínio cognitivo proposto por Brucki et al. ${ }^{14}$ considerando-se: 20 pontos para analfabetos; 25 pontos para pessoas com escolaridade de 1 a 4 anos; 26,5 para 5 a 8 anos; 28 para aqueles com 9 a 11 anos e 29 para mais de 11 anos.

A variável de exposição foi o comportamento sedentário, aferido por meio de duas questões que investigam o tempo que o idoso permanece sentado (em minutos e horas) durante suas atividades dentro ou fora de casa, as quais pertencem ao instrumento $I n-$ ternational Physical Activity Questionnaire (IPAQ), com validade para uso em idosos brasileiros ${ }^{15}$. O total do comportamento sedentário foi determinado com base na média ponderada do tempo sentado em um dia da semana e em um dia de final de semana [(tempo na semana $\times 5)+($ tempo no final de semana $\times 2)] / 7)^{16}$.Os idosos foram categorizados em quatro domínios: 1) 0 a 3 horas; 2) 4 a 7 horas; 3) 8 a 10 horas; e 4) 11 ou mais horas por $\mathrm{dia}^{17}$.

As variáveis de controle foram autorreferidas e incluídas separadamente no questionário do estudo, divididas em dois blocos: 1 ) bloco sociodemográfico, que incluiu sexo, faixa etária (60-69 anos; 70-79 anos; e 80 anos e mais), renda mensal ( $<1,5$ salários mínimos e $\geq 1,5$ salários mínimos), situação conjugal (casado/com companheiro; divorciado/solteiro e viúvo), cor da pele (branca; parda e outras); e 2) bloco estilo de vida, que avaliou o consumo de bebidas alcoólicas com base no instrumento The Alcohol Use Disorders Identification Test-AUDIT ${ }^{18}$ ( $\leq 1$ vez no mês; 2 a 4 vezes no mês; $\geq 2$ vezes na semana), e tabagismo (nunca fumou; fumou e parou; fuma atualmente).

As análises dos dados foram realizadas no software estatístico STATA versão 14.0 (Stata Corp, College Station, Texas, EUA). Nas análises descritivas foram calculadas as prevalências (\%) e respectivos intervalos de confiança de 95\% (IC95\%). Para verificar a associação entre o comportamento sedentário e o declínio cognitivo foram realizadas análises de Regressão Logística Multivariada, apresentando-se valores de odds ratio (OR) brutos e ajustados para as variáveis de controle, considerando-se dois modelos: 1) ajustado para variáveis sociodemográficas; 2) ajustado para variáveis sociodemográficas e de estilo de vida. Os valores de $\mathrm{p}$ $\leq 0,05$ foram considerados como estatisticamente significativos.

\section{Resultados}

Dentre os 540 idosos elegíveis, 308 foram avaliados conforme a descrição do fluxograma na Figura 1. Desses, 178 (57,8\%) eram do sexo feminino e tinham média de idade 60,8 anos ( $\mathrm{DP}=7,06$ anos). A maioria estudou até quatro anos $(48,6 \%)$, relatou ser casado ou viver com companheiro $(59,0 \%)$ e autodeclarou-se com cor da pele branca $(71,6 \%)$.

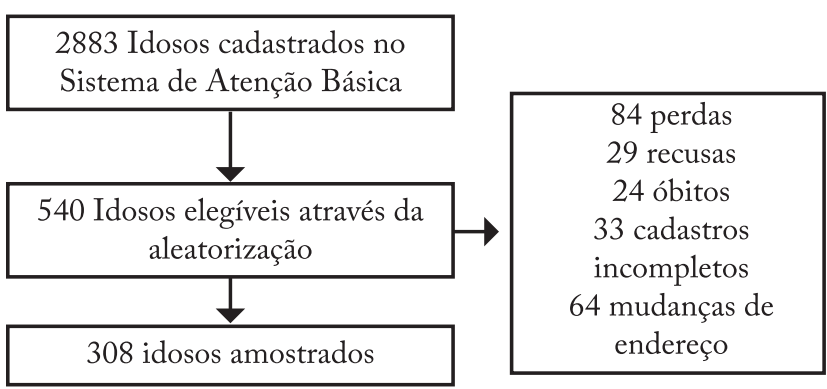

Figura 1 - Fluxograma dos idosos amostrados. Balneário Arroio do Silva, Santa Catarina, 2019.

Com relação ao comportamento sedentário, observou-se que a maioria dos idosos (53,0\%; IC95\%: 47,3; $58,4)$ relatou permanecer sentado até 3 horas em um dia de semana/final de semana, enquanto que $8,1 \%$ (IC95\%: 5,5; 11,7) permaneciam sentados 11 horas ou mais por dia. Maiores detalhes das características sociodemográficas e estilo de vida da amostra estão apresentados na Tabela 1 .

Em relação ao desfecho, 57,7\% (IC95\%: 51,6; 63,6) dos idosos analisados apresentaram declínio cognitivo, com proporções mais elevadas entre as mulheres (60,6\%), com 80 anos ou mais $(68,0 \%)$, viúvos $(65,0 \%)$, 
fumantes $(66,0 \%)$ e que ingeriam bebidas alcoólicas pelo menos uma vez no mês $(62,0 \%)$. O declínio cognitivo foi mais prevalente entre os idosos que permaneciam 11 horas ou mais sentados por dia $(89,0 \%)$, seguido daqueles que ficavam entre 8 e 10 horas nesse comportamento $(62,5 \%)$ - Tabela 1.

Considerando o modelo final da análise de regressão, os idosos que permaneciam 11 horas ou mais por dia na posição sentada tiveram cinco vezes maiores chances (OR = 5,03; IC95\%: 1,07; 23,61) de apresentarem declínio cognitivo quando comparados àqueles que permaneciam por até 3 horas nesse comportamen-

Tabela 1 - Descrição das prevalências de declínio cognitivo de acordo com características sociodemográficas e estilo de vida de idosos comunitários residentes em Balneário Arroio do Silva, Santa Catarina, Brasil, 2019.

\begin{tabular}{|c|c|c|}
\hline Características & $\mathrm{n}(\%)$ & $\begin{array}{c}\text { Declínio } \\
\text { cognitivo } \\
\% \text { (IC 95\%) }\end{array}$ \\
\hline \multicolumn{3}{|l|}{ Sexo $(n=308)$} \\
\hline Feminino & $178(57,8)$ & $60,6(52,5 ; 68,2)$ \\
\hline Masculino & $130(42,2)$ & $54,0(44,6 ; 63,0)$ \\
\hline \multicolumn{3}{|l|}{ Faixa etária $(\mathrm{n}=307)$} \\
\hline 60-69 anos & $168(54,7)$ & $60,5(52,4 ; 68,0)$ \\
\hline $70-79$ anos & $109(35,5)$ & $50,5(40,0 ; 61,1)$ \\
\hline$\geq 80$ anos & $30(9,8)$ & $68,0(46,2 ; 84,0)$ \\
\hline \multicolumn{3}{|l|}{ Situação conjugal $(\mathrm{n}=308)$} \\
\hline Casado/com companheiro & $182(59,0)$ & $55,2(47,4 ; 63,0)$ \\
\hline Divorciado/solteiro & $55(17,9)$ & $57,4(42,5 ; 71,1)$ \\
\hline Viúvo & $71(23,0)$ & $65,0(51,3 ; 76,4)$ \\
\hline \multicolumn{3}{|l|}{ Cor da pele $(\mathrm{n}=307)$} \\
\hline Branca & $220(71,7)$ & $55,4(48,3 ; 62,3)$ \\
\hline Parda & $66(21,5)$ & $60,0(46,2 ; 72,3)$ \\
\hline Outras & $21(6,9)$ & $81,2(51,4 ; 94,6)$ \\
\hline \multicolumn{3}{|l|}{ Renda mensal $(\mathrm{n}=305)$} \\
\hline$<1,5$ salários mínimos & $173(56,7)$ & $59,7(51,4 ; 67,5)$ \\
\hline$\geq 1,5$ salários mínimos & $132(43,3)$ & $56,7(47,5 ; 65,5)$ \\
\hline \multicolumn{3}{|l|}{ Tabagismo $(\mathrm{n}=307)$} \\
\hline Nunca fumou & $136(44,3)$ & $60,0(50,5 ; 68,4)$ \\
\hline Fumou e parou & $121(39,4)$ & $53,0(43,2 ; 62,2)$ \\
\hline Fuma atualmente & $50(16,3)$ & $66,0(49,5 ; 79,0)$ \\
\hline \multicolumn{3}{|l|}{ Uso de álcool $(\mathrm{n}=307)$} \\
\hline Nunca & $199(64,9)$ & $58,1(50,4 ; 65,5)$ \\
\hline$\leq 1$ vez no mês & $47(15,3)$ & $62,0(46,0 ; 75,6)$ \\
\hline 2 a 4 vezes no mês & $32(10,4)$ & $48,2(30,0 ; 67,0)$ \\
\hline$\geq 2$ vezes na semana & $29(9,4)$ & $57,1(37,5 ; 74,7)$ \\
\hline \multicolumn{3}{|c|}{ Comportamento sedentário $(n=308)$} \\
\hline 0-3 horas & $163(53,0)$ & $57,7(49,3 ; 65,6)$ \\
\hline 4-7 horas & $100(32,4)$ & $50,5(40,1 ; 61,0)$ \\
\hline 8-10 horas & $20(6,5)$ & $62,5(35,0 ; 84,0)$ \\
\hline 11 ou mais horas & $25(8,1)$ & $89,0(61,0 ; 97,6)$ \\
\hline
\end{tabular}

to, independente das características sociodemográficas e estilo de vida (Tabela 2).

\section{Discussão}

Os principais resultados deste estudo evidenciaram que os idosos que permaneciam 11 ou mais horas por dia em comportamento sedentário apresentaram cinco vezes mais chances de declínio cognitivo, em comparação aos que ficavam até 3 horas nesse comportamento.

Em relação às prevalências observadas de declínio cognitivo, nossos resultados assemelham-se aos de outros estudos brasileiros, destacando-se maior ocorrência no sexo feminino ${ }^{4,7,19}$. Acredita-se que essa maior prevalência em idosas possa ser justificada pela mortalidade diferenciada entre os sexos, com predomínio de maior mortalidade em homens, especialmente na população idosa brasileira ${ }^{19}$. As mulheres constituem a maior parte da população idosa e vivem em média sete anos mais do que os homens; assim observa-se a feminização do envelhecimento em território nacional ${ }^{20}$. Outro fator importante é a menopausa, que envolve alterações hormonais passíveis de afetar o funcionamento do cérebro e a cognição, devido à mudança nos níveis de estrogênio circulantes no organismo ${ }^{21}$.

A idade também é um dos fatores determinantes para o declínio cognitivo em idosos ${ }^{22}$. Concordantes aos nossos achados, a literatura apresenta que idosos com idade superior a 80 anos apresentam maiores prevalências de declínio cognitivo ${ }^{4,6,7}$. Considera-se que os idosos com mais idade sofrem maiores alterações biológicas, psicológicas e sociais, as quais podem gerar comprometimentos nos mecanismos de aprendizagem, memória e cognição, especialmente nas tarefas que exigem rapidez, atenção, concentração e raciocínio indutivo. Com o avanço da idade, o declínio cognitivo também pode aparecer em decorrência das degenerações nas funções e capacidades cerebrais, de modo que a plasticidade, a codificação, o armazenamento e a recuperação de informações se tornam menos eficientes ou são interrompidas devido à redução da atenção e da memória de curto prazo ${ }^{23}$.

A presença de um companheiro pode se constituir como um apoio para as dificuldades ocasionadas pelo declínio cognitivo e também no incentivo à busca dos cuidados necessários à manutenção da saúde ${ }^{24}$. Assim nossos achados concordam com a literatura que sugere que os idosos que moram sem o cônjuge apresentam piora na cogniçãao ${ }^{7,21,22,24}$, principalmente pela perda de suporte social. Referente ao estilo de vida, nossos acha- 
Tabela 2 - Modelos de análise de Regressão Logística Multivariada entre a presença de declínio cognitivo e o comportamento sedentário de idosos comunitários, residentes em Balneário Arroio do Silva, Santa Catarina, Brasil, 2019.

\begin{tabular}{|c|c|c|c|}
\hline \multirow[b]{2}{*}{ Características } & \multicolumn{3}{|c|}{ Declínio cognitivo } \\
\hline & $\begin{array}{l}\text { Análise Bruta } \\
\text { OR (IC 95\%) }\end{array}$ & $\begin{array}{c}\text { Modelo } 1 \\
\text { OR (IC 95\%) }\end{array}$ & $\begin{array}{c}\text { Modelo 2 } \\
\text { OR (IC 95\%) }\end{array}$ \\
\hline \multicolumn{4}{|l|}{ Comportamento sedentário } \\
\hline 0-3 horas & 1,00 & 1,00 & 1,00 \\
\hline 4-7 horas & $0,75(0,44 ; 1,27)$ & $0,75(0,43 ; 1,31)$ & $0,78(0,44 ; 1,35)$ \\
\hline $8-10$ horas & $1,22(0,42 ; 3,54)$ & $1,80(0,52 ; 6,16)$ & $1,78(0,40 ; 6,48)$ \\
\hline 11 ou mais horas & $5,85(1,29 ; 26,4)^{*}$ & $5,00(1,07 ; 23,29)^{*}$ & $5,03(1,07 ; 23,61)^{*}$ \\
\hline \multicolumn{4}{|l|}{ Sexo } \\
\hline Feminino & 1,00 & 1,00 & 1,00 \\
\hline Masculino & $0,76(0,46 ; 1,24)$ & $0,89(0,51 ; 1,55)$ & $0,93(0,50 ; 1,70)$ \\
\hline \multicolumn{4}{|l|}{ Faixa etária } \\
\hline 60-69 anos & 1,00 & 1,00 & 1,00 \\
\hline 70-79 anos & $0,67(0,39 ; 1,35)$ & $0,59(0,33 ; 1,03)$ & $0,59(0,32 ; 1,05)$ \\
\hline$\geq 80$ anos & $1,38(0,56 ; 3,41)$ & $1,09(0,42 ; 2,84)$ & $1,06(0,40 ; 2,83)$ \\
\hline \multicolumn{4}{|l|}{ Situação conjugal } \\
\hline Casado/com companheiro & 1,00 & 1,00 & 1,00 \\
\hline Divorciado/solteiro & $1,09(0,56 ; 2,10)$ & $1,04(0,52 ; 2,07)$ & $1,03(0,50 ; 2,11)$ \\
\hline Viúvo & $1,50(0,80 ; 2,80)$ & $1,30(0,62 ; 2,67)$ & $1,33(0,63 ; 2,80)$ \\
\hline \multicolumn{4}{|l|}{ Cor da pele } \\
\hline Branca & 1,00 & 1,00 & 1,00 \\
\hline Parda & $1,20(0,65 ; 2,21)$ & $1,12(0,60 ; 2,11)$ & $1,20(0,62 ; 2,31)$ \\
\hline Outras & $3,48(0,96 ; 12,61)$ & $3,50(0,92 ; 13,24)$ & $3,82(0,99 ; 14,77)$ \\
\hline \multicolumn{4}{|l|}{ Renda mensal } \\
\hline$<1,5$ salários mínimos & 1,00 & 1,00 & 1,00 \\
\hline$\geq 1,5$ salários mínimos & $0,89(0,54 ; 1,45)$ & $0,92(0,53 ; 1,60)$ & $1,02(0,58 ; 1,82)$ \\
\hline \multicolumn{4}{|l|}{ Tabagismo } \\
\hline Nunca fumou & 1,00 & - & \\
\hline Fumou e parou & $0,72(0,44 ; 1,27)$ & - & $0,83(0,45 ; 1,50)$ \\
\hline Fuma atualmente & $1,30(0,61 ; 2,72)$ & - & $1,04(0,45 ; 2,40)$ \\
\hline \multicolumn{4}{|l|}{ Uso de álcool } \\
\hline Nunca & 1,00 & - & 1,00 \\
\hline$\leq 1$ vez no mês & $1,17(0,60 ; 2,34)$ & - & $1,10(0,52 ; 2,30)$ \\
\hline 2 a 4 vezes no mês & $0,67(0,30 ; 1,48)$ & - & $0,61(0,25 ; 1,50)$ \\
\hline$\geq 2$ vezes na semana & $0,95(0,42 ; 2,15)$ & - & $1,08(0,43 ; 2,73)$ \\
\hline
\end{tabular}

*Associação estatisticamente significativa; Modelo 1 = ajustado para características sociodemográficas; Modelo 2 = ajustado para características sociodemográficas e estilo de vida; OR = odds ratio; IC95\% = Intervalo de confiança de 95\%.

dos demonstraram que, dentre os idosos com declínio cognitivo, a maioria fumava e ingeria bebidas alcóolicas ao menos uma vez no mês. Considera-se que o hábito de fumar e o etilismo podem predispor às doenças crônicas, interferir em medicações e acelerar a perda das habilidades funcionais, as quais poderiam contribuir para o declínio cognitivo a longo prazo ${ }^{25}$.

Nossos principais achados indicaram que os idosos que passavam maior tempo do seu dia em comportamento sedentário (11 ou mais horas) tiveram as maiores chances de declínio cognitivo. Acredita-se que despender maior tempo diário em atividades que requerem gasto energético acima dos níveis basais pode contribuir para o aumento substancial da vascularização, e, consequentemente, da oxigenação cerebral e da área cognitiva ativa ${ }^{26}$.

Além disso, em uma revisão sistemática ${ }^{27}$ envolvendo 2.269 pacientes com demência, foi verificado que o comportamento sedentário prolongado pode prejudicar o metabolismo da glicose e dos lipídios, contribuindo para o declínio cognitivo e demência. Sugeriu-se, ainda, que o comportamento sedentário está relacionado ao 
maior volume de hiperintensidade da substância branca, um biomarcador para o risco de declínio cognitivo.

Em estudo transversal composto por 310 idosos residentes no município de Ibicuí-Bahia, constatou-se que o excesso de tempo em comportamento sedentário foi um indicador válido para identificar transtornos mentais comuns, incluindo o declínio cognitivo. Os autores reforçam que esse comportamento deve ser observado como um fator de risco importante para a perda cognitiva pelos profissionais de saúde que atuam com os idosos ${ }^{25}$.Ainda, no estudo realizado com idosos de Viçosa-Minas Gerais ${ }^{26}$, embora os resultados não tenham sido significativos, os autores observaram que aqueles que praticavam menos atividades físicas tiveram maiores valores nas razões de chances brutas e ajustadas para a presença de declínio cognitivo.

Em revisão sistemática de evidências observacionais com objetivo de avaliar a associação entre comportamento sedentário e função cognitiva ${ }^{8}$, os autores concluíram que o aumento do comportamento sedentário está associado a menor função cognitiva. Porém, apenas oito estudos foram incluídos e a heterogeneidade dos mesmos impediu o cálculo da magnitude dessa associação. Em outro estudo realizado com população idosa chilena ${ }^{28}$, verificou-se que o maior tempo gasto sentado foi positivamente associado à suspeita de declínio cognitivo. Os participantes que relataram permanecer mais de 8 horas/dia sentados apresentaram chances elevadas de declínio cognitivo em comparação aos que permaneciam menos de 4 horas/dia.

Diante desses achados, torna-se importante ressaltar que, independentemente do tempo de realização de atividades físicas, os idosos que passam mais de 8 horas sentados por dia têm associação com declínio cogniti$\mathrm{vo}^{23}$. Apesar disso, não há, ainda, ponto de corte na literatura que discrimine a quantidade exata de qual o tempo exposto ao comportamento sedentário seria capaz de causar agravos para a saúde conforme a faixa etária.

Entretanto, vale ressaltar que nem todos os comportamentos sedentários são iguais, sendo que a televisão, que responde por mais da metade de todas as atividades de lazer, pode apresentar o maior risco devido ao menor gasto de energia $(\mathrm{MET}=1,0) \mathrm{e}$, possivelmente, levar ao menor engajamento cognitivo do que outros comportamentos sedentários mais cognitivamente estimulantes, tais como ler e digitar (METs $=1,8)$, tocar piano $(\mathrm{METs}=2,5)$ ou jogar jogos de tabuleiro $(\mathrm{METs}=1,5)^{29}$. Entende-se que interrupções no comportamento sedentário são extremamente be- néficas e reduzem os riscos para o declínio cognitivo, sugerindo-se que seja considerada a redução do tempo sedentário discricionário para $<2 \mathrm{~h} /$ dia, e envolvimento concomitante de $\geq 150 \mathrm{~min} / \mathrm{semana}$ de atividade física moderada a vigorosa para se alcançar o envelhecimento cognitivo saudável ${ }^{8}$.

Algumas limitações do presente estudo precisam ser mencionadas. Primeiro, a avaliação única (estudo transversal) da amostra não permite determinar as relações de causa e efeito entre as variáveis estudadas, podendo-se, apenas, estimar as chances de associação entre as mesmas. Segundo, os dados sobre comportamento sedentário foram autorrelatados e avaliados por apenas duas questões sobre o tempo dispendido em posição sentada, o que pode ter levado à sub ou sobre estimação desse comportamento. Vale ressaltar que uma revisão sistemática publicada recentemente forneceu evidências inconsistentes de como o comportamento sedentário em idosos estão sendo caracterizados, mostrando que não há padronização dos métodos empregados para sua avaliação e que, por isso, comparar esses resultados se torna uma tarefa difícil ${ }^{30}$. E ainda, a avaliação do declínio cognitivo foi realizada pelo MEEM, o qual apresenta diferentes pontos de cortes estabelecidos na literatura, sem consenso do melhor e/ ou mais fidedigno para classificar corretamente os idosos com essa condição.

Apesar disso, considera-se o fato de que os instrumentos empregados para a avaliação do comportamento sedentário e do declínio cognitivo são extensamente utilizados na literatura, não apenas no Brasil, mas também em diferentes países, o que favorece a comparação dos resultados encontrados com futuros estudos. A representatividade da amostra avaliada no presente estudo também é um ponto positivo a ser ressaltado, uma vez que seu cálculo considerou todos os idosos que utilizavam as Unidades Básicas de Saúde do município, e seus resultados podem contribuir para subsidiar propostas de intervenções que contemplem as ações de saúde desenvolvidas na Atenção Básica.

Em conclusão, observou-se associação positiva entre comportamento sedentário e declínio cognitivo, na qual idosos que permaneciam 11 ou mais horas sentados por dia tiveram maiores chances de terem declínio cognitivo quando comparados àqueles que relataram permanecer até três horas nesse comportamento. Além disso, a função cognitiva é um dos componentes mais importantes do envelhecimento saudável e está diretamente ligada à realização das atividades do cotidiano 
dos idosos, o que a torna um objetivo cada vez mais relevante para as investigações epidemiológicas que indicam que sua promoção pode ser alcançada por meio de mudanças de comportamentos.

\section{Conflito de interesse}

Os autores declaram não haver conflito de interesse.

\section{Contribuição dos autores}

Horacio PR participou na concepção, planejamento, interpretação dos dados, revisão bibliográfica, escrita e revisão final do manuscrito. Avelar NCP participou na interpretação dos dados, revisão bibliográfica e revisão final do manuscrito. Danielewicz AL participou na concepção, planejamento, análise e interpretação dos dados, escrita do trabalho e revisão final do manuscrito.

\section{Agradecimentos}

Os autores agradecem a Secretaria Municipal de Saúde e os profissionais que atuam nas Unidades Básicas de Saúde do município Balneário Arroio do Silva de Santa Catarina por auxiliarem na realização do projeto e viabilização do contato com os idosos amostrados.

\section{Referências}

1. Oliveira DV, Lima MCC, Oliveira GVN, Bertolini SMMG, Nascimento JRA, Cavaglieri CR. Is sedentary behavior an intervening factor in the practice of physical activity in the elderly? Rev Bras Geriatr e Gerontol. 2018; 21(4):472-79.

2. Alzheimer's Association and Centers for Disease Control and Prevention. The Healthy Brain Initiative: The Public Health Road Map for State and National Partnerships, 2013-2018: Chicago, IL: Alzheimer's Association. 2013. Disponível em: https://www.cdc.gov/aging/pdf/2013-healthy-braininitiative.pdf [agosto de 2020].

3. Overton M, Pihlsgård M, Elmståhl S: Prevalence and Incidence of Mild Cognitive Impairment across Subtypes, Age, and Sex. Dement Geriatr Cogn Disord. 2019;47:219-32.

4. Faria EC, Silva SA, Farias KRA, Cintra A. Avaliação cognitiva de pessoas idosas cadastradas na estratégia saúde da família: município do Sul de Minas. Rev da Esc Enferm da USP. 2011;45(2):1748-52.

5. Ferreira RC, Duarte AMV, Fernandes NCN, Souza JGS, Sá MAB, Oliveira LFB. et al. O idoso com comprometimento cognitivo apresenta pior condição de saúde bucal? Cienc e Saude Coletiva. 2014;19(8):3417-28.

6. Holz AW, Nunes BP, Thumé E, Lange C, Facchini LA. Prevalence of cognitive impairment and associated factors among the elderly in Bagé, Rio Grande do Sul, Brazil. Rev Bras Epidemiol. 2013;16(4):880-8.

7. Nascimento RASA, Batista RTS, Rocha SV, Vasconcelos LRC. Prevalência e fatores associados ao declínio cognitivo em idosos com baixa condição econômica: Estudo MONIDI. J Bras Psiquiatr. 2015;64(3):187-92.

8. Falck RS, Davis JC, Liu-Ambrose T. What is the association between sedentary behaviour and cognitive function? A systematic review. Br J Sports Med. 2017;51(10):800-11.
9. Antunes HKM, Santos RF, Cassilhas R, Santos RVT, Bueno OFA, Mello MT. Exercício físico e função cognitiva: Uma revisão. Rev Bras Med do Esporte. 2006;12(2):108-14.

10. Harvey JA, Chastin SFM, Skelton DA. How Sedentary Are Older People? A Systematic Review of the Amount of Sedentary Behavior. J Aging Phys Act. 2015;23(3):471-87.

11. IBGE. Projeções e estimativas da população do Brasil e das Unidades da Federação: 2010. Disponível em: https://www. ibge.gov.br/apps/populacao/projecao/ [Agosto de 2020].

12. PNUD - Programa das Nações Unidas para o Desenvolvimento. İndice de Desenvolvimento Humano Municipal 2010. Disponível em: https://www.br.undp.org/ content/brazil/pt/home/idh0/rankings/idhm-municipios2010[Agosto de 2020].

13. Bertolucci PHF, Brucki SMD, Campacci SR, Juliano Y. O Mini-Exame do Estado Mental em uma população geral: impacto da escolaridade. Arq Neuropsiquiatr. 1994;52(1):01-7.

14. Brucki SMD, Nitrin R, Caramelli P, Bertolucci PHF, Okamoto IH. Sugestões para o uso do mini-exame do estado mental no Brasil. Arq Neuropsiquiatr. 2003;61(3):777-81.

15. Benedetti TB, Mazo GZ, Barros MVG. Aplicação do Questionário Internacional de Atividades Físicas para avaliação do nível de atividades físicas de mulheres idosas: validade concorrente e reprodutibilidade teste-reteste. RevBrasCiênc Mov. 2004;12:25-34.

16. Silva RDC, Meneguci J, Martins TI, Santos AS, Sasaki JE, Tribess S, et al. Associação entre tempo sentado e diabetes mellitus em idosos: Um estudo de base populacional. Rev Bras Cineantropometria e Desempenho Hum. 2015;17(4):379-88.

17. Smith L, Tully M, Jacob L, Blackburn N, Adlakha D, Caserotti P, et al. The Association Between Sedentary Behavior and Sarcopenia Among Adults Aged $\geq 65$ Years in Low- and Middle-Income Countries. Int J Environ Res Public Health. 2020;17(5):1708.

18. Babor TF, Higgins-Biddle JC, Saunders JB, Monteiro MG. The alcohol use disorders identification test: Guidelines for use in. World Health Organization, 2001.

19. Santos SR, Santos IBC, Fernandes MGM, Henriques MERM. Qualidade de vida do idoso na comunidade: aplicação da escala de Flanagan. Rev Lat Am Enfermagem. 2002;10(6):757-64.

20. Lebrão ML, Laurenti R. Saúde, bem-estar e envelhecimento: o estudo SABE no Município de São Paulo. Rev Bras Epidemiol. 2005;8(2):127-41.

21. Dumas, JA. Strategies for preventing cognitive decline in healthy older adults. The Canadian Journal of Psychiatry, 2017;62(11), 754-760.

22. Valle EA, Castro-Costa E, FirmoJOA, Uchoa E, Lima-Costa MF. Estudo de base populacional dos fatores associados ao desempenho no Mini Exame do Estado Mental entre idosos: Projeto Bambuí. Cad Saude Publica. 2009;25(4):918-26.

23. Williams KN, Kemper S. Interventions to reduce cognitive dfecline in aging. J. Psychosoc. Nurs Mental Health Serv. 2010;48(5):42-51.

24. Nunes WA, Dias FA, Nascimento JS, Gomes NC, Tavares DMS. Cognition, functionality and depression indicative among elderly. Rev da Rede Enferm do Nord. 2016;17(1):103-11.

25. Silva PAS, Rocha SV, Vasconcelos LRC, Santos CA. Comportamento sedentário como discriminador dos transtornos mentais comuns em idosos. J Bras Psiquiatr. 2017;66(4):183-8. 
26. Machado JC, Ribeiro RCL, Cotta RMM, Leal PFG. Declínio cognitivo de idosos e sua associação com fatores epidemiológicos em Viçosa, Minas Gerais. Rev Bras Geriatr e Gerontol. 2011;14(1):109-21.

27. Olanrewaju, O, Stockwell, S, Stubbs, B, \& Smith, L. Sedentary behaviours, cognitive function, and possible mechanisms in older adults: A systematic review. Aging clinical and experimental research, 2020;32(6):969-84.

28. Poblete-Valderrama F, Rivera CF, Petermann-Rocha F, Leiva AM, Martínez-Sanguinetti MA, Troncoso C, Mardones L,Villagrán M, Nazar G, Ulloa N, Martorell M, et al. Actividad física y tiempo sedente se asocian a sospecha de deterioro cognitivo en población adulta mayor chilena. Rev Med Chile. 2019;147(10):1247-55.
29. Katzmarzyk PT, Powell KE, Jakicic JM, Troiano RP, Piercy K, Tennant B. Sedentary Behavior and Health: Update from the 2018 Physical Activity Guidelines Advisory Committee. Med Sci Sports Exerc. 2019;51(6):1227-41.

30. Lins-Filho OL, Braga MM, Lima TM, Ferreira DKS. Low level of physical activity and sedentary behaviour in elderly: a systematic review of the parameters. Rev Bras Cineantropom Desempenho Hum. 2020;22:e60810.

Recebido: 30/09/2020

Aprovado: 05/03/2021

\section{Como citar este artigo:}

